\title{
MIMO-OFDM equaliser for spatial multiplexing transmission modes
}

\author{
P. Beinschob and U. Zölzer \\ Department of Signal Processing and Communications, Helmut-Schmidt-Universität/University of the Federal Armed Forces \\ Hamburg, Holstenhofweg 85, 22043 Hamburg, Germany
}

\begin{abstract}
In search for faster and more reliable communication, multiple-input multiple-output (MIMO) in conjuction with Orthogonal Frequency Division Multiplexing (OFDM) are subject of extensive research. In spatial multiplexing transmission an instantaneous rise of data rates governed by the number of transmit antennas can be realised. The system performance depends highly on signal-to-interference-plusnoise ratios (SINR) at the receiver. The receiver's equaliser is supposed to maximize the SINR by mitigating the spatial interference and thus separating the transmitted signals. For this problem several solutions exist such as linear and nonlinear, per subcarrier or OFDM symbol-based. An overview of common algorithms is given and complexity is discussed. Bit error rate (BER) performance evaluations are presented. Another aspect is the impact of the equalisation strategy on the performance of bit-interleaved soft information-based channel coding schemes. As a representative, LDPC codes are chosen. Simulation results show a significant BER performance loss for symbol decision-based equalisers compared to the uncoded performance. To overcome this problem a modification of the Maximum Likelihood algorithm is proposed which yields good performance for low SNR applications.
\end{abstract}

\section{Introduction}

In upcoming MIMO OFDM systems Spatial Multiplexing is an convenient way to linearly increase bandwidth efficiency with the number of transmit and receive antennas primary at the expense of higher SNR demands. Detection algorithms

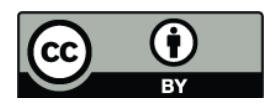

Correspondence to: $\mathrm{P}$. Beinschob (patric.beinschob@hsu-hamburg.de) tend to vary in complexity, the lower the SNR requirements the higher the complexity.

Mobile scenarios are a particular challenge. This is partly due to outdated channel state information (CSI) as well as the fact that many receivers neglect the time-variant behaviour of the channel during a frame and even OFDM symbol. The multiple, independent OFDM send signals are superposed in the wireless channel, modelled by a channel matrix and additive, white gaussian noise (AWGN). The signals are convoluted by a time-varying channel matrix that results in a nonlinear system model. Thus, orthogonality of the OFDM subcarriers is disturbed. Simulation results based on 3GPP MIMO Spatial Channel Model show the detection performance over SNR for a range of velocities.

The rest of the paper is organized as follows. The underlying system model in time and frequency-domain, as well as a vectorised representation are presented in Sect. 2, followed by a brief review of per layer MIMO detection algorithms in Sect. 3. Joint detectors are discussed in Sect. 4. Furthermore the proposed time-domain interference mitigation is presented in Sect. 5. The paper is concluded by illustrating simulation results in Sect. 6 and a conclusion.

\section{System model and structure}

The vector of received values $\mathbf{r}$ at the time sample $m$ of a MIMO system is the superposition of $L \cdot n_{T}$ previously sent samples and the current $n_{T}$ samples, where $L+1$ is the length of the sampled channel impulse response and $n_{T}$ is the number of transmit antennas. It is given by

$\mathbf{r}[m]=\sum_{l=0}^{L} \mathbf{h}[l, m] \cdot \mathbf{s}[m-l]+\tilde{\mathbf{w}}[m]$,

where $\mathbf{s}[m]$ denotes the current vector of symbols of each of the transmit antenna, $\mathbf{w}$ is an identically, independently dis-

Published by Copernicus Publications on behalf of the URSI Landesausschuss in der Bundesrepublik Deutschland e.V. 

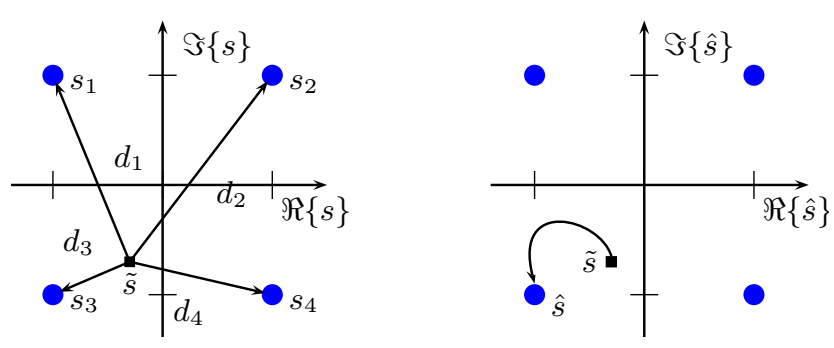

Fig. 1. Per layer maximum likelihood symbol decision and quantization.

tributed (iid) additive white Gaussian noise term and $\mathbf{h}[l, m]$ is the MIMO channel matrix in delay and time domain, indexed with $l$ respectively $m$. The past sent samples are denoted by $\mathbf{s}[m-l]$, for $l \neq 0, l \leq L$. The data symbols of the $K$ subcarriers are modulated by an inverse Fast Fourier Transform (IFFT). In simulations every value corresponding to a transmit antenna of the resulting vectors is transmitted using the formula above. The data symbols itself are drawn from an $M$-order QAM modulation alphabet $\mathcal{M}$. The mapping, denoted by $\mathcal{M}\{\cdot\}$, modulates $\kappa=\log _{2} M$ bits to a QAM symbol. This is done consecutively for all $n_{T}$ send streams/layer. The QAM constellations are considered power-normalized to simplify notation.

In frequency domain the system model in Eq. (1) can be described as

$\mathbf{r}[n, k]=\mathbf{H}[n, k] \cdot \mathbf{s}[n, k]+\mathbf{w}[n, k]$,

where $n$ denotes the time index of an OFDM symbol and $k$ its subcarrier index. The vectors $\mathbf{r}[n, k]$ and $\mathbf{w}[n, k]$ are of dimension $n_{R} \times 1, \mathbf{s}[n, k]$ of $n_{T} \times 1$ and the matrix $\mathbf{H}[n, k]$ of $n_{R} \times n_{T}$, at which $n_{R}$ is the number of transmit antennas. In simulations the MIMO channel coefficients $H_{r, t}[n, k], r=$ $1, \ldots, n_{R}, t=1, \ldots, n_{T}$ are modeled using the $3 \mathrm{GPP}$ spatial model which was developed to evaluate receiver algorithms in MIMO scenarios (3rd Generation Partnership Project 3GPP-TR25.996, 2008). The superposed received signals are transferred back into the frequency domain with the help of a FFT, resulting in the vectors $\mathbf{r}[n, k]$ of Eq. (2). A perfectly known channel matrix $\mathbf{H}$ is assumed for all detectors, as well as perfect synchronization and total avoidance of block interference, i.e. the cyclic prefix is longer than the maximum delay path. The MIMO detector's performance is evaluated in terms of soft information in form of channel loglikelihood ratios (LLRs), $L_{C}(x)$ and in direct form as hard decided bits.

\subsection{Vectorised system model}

The vectorised system description of Eq. (1) - for a block symbol based system where block interference is avoided, e.g. OFDM with cyclic prefix removed - can be denotes as

$\mathbf{r}^{(\mathbf{v})}=\mathbf{h}^{(\mathbf{v})} \cdot \mathbf{s}^{(\mathbf{v})}+\mathbf{w}^{(\mathbf{v})}$, where the dimensions are $\mathbf{r}^{(\mathbf{v})}, \mathbf{w}^{(\mathbf{v})} \in \mathbb{C}^{K \cdot n_{R}}, \mathbf{s}^{(\mathbf{v})} \in \mathbb{C}^{K \cdot n_{T}}$ and $\mathbf{h}^{(\mathbf{v})} \in \mathbb{C}^{K \cdot n_{R} \times K \cdot n_{T}}$, (Stamoulis et al., 2002). The channel matrix structure is as follows:

$\mathbf{h}^{(\mathbf{v})}=\left(\begin{array}{ccccc}\mathbf{h}[0,0] & \mathbf{0} & & \cdots & \mathbf{0} \\ \mathbf{h}[1,0] & \mathbf{h}[0,1] & \mathbf{0} & \cdots & \\ \vdots & \vdots & \mathbf{h}[0, m] & \cdots & \\ \mathbf{h}[l, 0] & \mathbf{h}[l-1,1] & \ddots & \ddots & \\ \vdots & \vdots & & \ddots & \\ \mathbf{h}[L+1,0] & \mathbf{h}[L, 1] & & \ddots & \vdots \\ \mathbf{0} & \mathbf{h}[L+1,1] & \cdots & \ddots & \\ \vdots & \vdots & & \ddots & \mathbf{0} \\ \mathbf{0} & \mathbf{0} & \cdots & \cdots & \mathbf{h}[0, K]\end{array}\right)$,

where

$$
\begin{aligned}
\mathbf{h}[l, m]= & \left(\begin{array}{ccc}
h_{1,1}[l, m] & \ldots & h_{1, n_{T}}[l, m] \\
\vdots & \ddots & \vdots \\
h_{n_{R}, 1}[l, m] & \ldots & h_{n_{R}, n_{T}}[l, m]
\end{array}\right), \\
& 0 \leq l \leq L, \quad 0 \leq m \leq K .
\end{aligned}
$$

The vectorised formulation of the send vector is as follows

$\mathbf{s}^{(\mathbf{v})}=\left(s_{1}[1], \ldots, s_{n_{T}}[1], \ldots, s_{1}[m], \ldots, s_{n_{T}}[K],\right)^{T}$.

Analogue for the receive vector

$\mathbf{r}^{(\mathbf{v})}=\left(r_{1}[1], \ldots, r_{n_{R}}[1], \ldots, r_{1}[m], \ldots, r_{n_{R}}[K]\right)^{T}$.

\section{Per layer detection}

The idea of MIMO per layer detection is to map the problem back into the send space and decide upon the most likely symbol per send layer or send antenna stream $t=1, \ldots, n_{T}$ - which is identical in spatial multiplexing - as depicted in Fig. 1. This is reduction in complexity but leads to suboptimal results compared to joint layer detection.

\subsection{Linear receivers}

Zero-Forcing is well known method, an optimal linear solution neglecting noise is given by

$\tilde{\mathbf{s}}=\left(\mathbf{H}^{H} \mathbf{H}\right)^{-1} \mathbf{H}^{H} \mathbf{r}$.

High amplification of noise for low singular values leads to bad performance. The complexity for a block symbol is $\mathcal{O}\left(K n_{R} n_{T}^{2}\right)$. If noise is considered, the so-called linear MMSE solution is obtained,

$\tilde{\mathbf{s}}=\left(\mathbf{H}^{H} \mathbf{H}+\sigma_{w}^{2} \mathbf{I}_{n_{R}}\right)^{-1} \mathbf{H}^{H} \mathbf{r}$

It is limiting amplification, thus power, with a complexity of $\mathcal{O}\left(K n_{R} n_{T}^{2}\right)$. 


\subsection{Successive interference cancelation}

Traditional Zero-Forcing approach is hard-decision only with a complexity of $\mathcal{O}\left(K n_{R}^{2} n_{T}^{2}\right)$ (Wolniansky et al., 1998). The following improved algorithms are also considered here: the VBLAST/MMSE variant, which utilize Eq. (9) for cancelation and a maximum a-posteriori order criterion is developed by Yapici (2005), called VBLAST/ZF/MAP.

\section{Joint layer detection}

\subsection{Maximum likelihood symbol vector detection}

Based upon $\mathbf{r}$ and channel matrix knowledge $\mathbf{H}$ optimal detection in a Maximum Likelihood (ML) sense is determining the most probable send symbol vector $\mathbf{s}$, minimizing the distance:

$\operatorname{argmin}_{\hat{\mathbf{s}}}\|\mathbf{r}-\mathbf{H} \cdot \hat{\mathbf{s}}\|^{2} \quad \forall \hat{\mathbf{s}} \in \mathcal{S}$,

where $\mathcal{S}=\mathcal{M}^{n_{T}}$ is the set of all possible send vectors. It contains $M^{n_{T}}$ vectors. Testing all combinations and taking the most probable, yields optimal results at exponential complexity $O\left(K M^{n_{T}}\right)$. A well investigated approach to reduce the complexity by maintaining Maximum-Likelihood performance is the so called Sphere Decoding (Hochwald and ten Brink, 2003).

\subsection{Soft maximum likelihood detector}

Realizing Eq. (10) straight forward, a list of all $M^{n_{T}}$ distances is created:

$d_{i}^{2}=\left\|\mathbf{r}-\mathbf{H} \cdot \hat{\mathbf{s}}_{i}\right\|^{2}$.

Instead of considering only $\hat{\mathbf{s}}_{j}$ with $d_{j}=\min _{i} d_{i}$ values, the neighborhood of the minimum is incorporated in the detection. Basic idea is to consider hypothetic vectors in an expectation value within a radius around $\mathbf{r}$ assuming Gaussian noise:

$$
\begin{aligned}
\tilde{\mathbf{s}} & =\sum_{j \in S_{\sigma}} p_{j} \mathbf{s}_{j}, S_{\sigma}=\left\{i \in\left\{1, \ldots, M^{n_{T}}\right\} \mid d_{i}<c \cdot \sigma_{w}\right\}, \\
p_{j} & =\exp \left(-d_{j}^{2} / \sigma_{w}^{2}\right) / \sum_{S_{\sigma}} \exp \left(-d_{j}^{2} / \sigma_{w}^{2}\right)
\end{aligned}
$$

The factor $c$ of the noise deviation $\sigma_{w}$ can be varied, it is set to $c:=6$ in the following. The complexity is the same as in the ML case.

\section{Time domain interference mitigation}

For time domain equalisation (TEQ), the vectorised system description Eq. (3) is considered. The Discrete Fourier Transform (DFT) matrix for the vectorised MIMO system can be written as $\mathbf{W}=\tilde{\mathbf{W}} \otimes \mathbf{I}_{n_{T}}$ with $\tilde{\mathbf{W}}_{k, m}=$
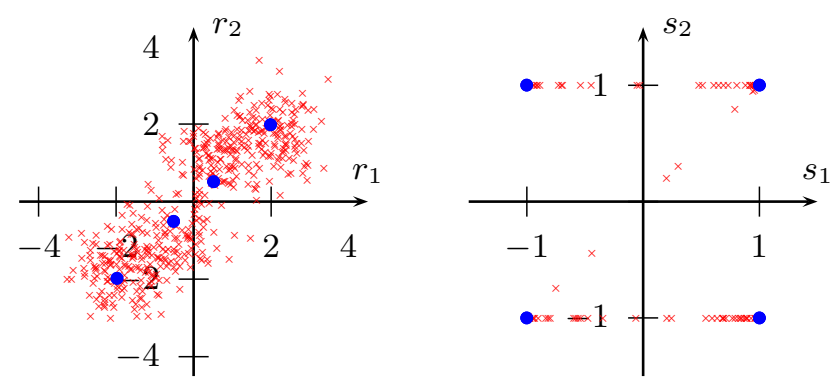

Fig. 2. Receive and send vector space for a BPSK $2 \times 2$ system with proposed SoftML equaliser.

$\frac{1}{\sqrt{K}} e^{-j(2 \pi / K) k m}$. The operator $\otimes$ denotes the Kronecker product. Following the MMSE criterion, an optimal interference mitigating filter matrix $\Psi$ maximizing SINR (Stamoulis et al., 2002), is calculated by

$$
\begin{aligned}
\mathbf{R}_{\mathbf{h h}} & =\sigma_{N} \mathbf{I}_{K \cdot n_{R}}+\mathbf{h}^{(\mathbf{v})} \cdot\left(\mathbf{h}^{(\mathbf{v})}\right)^{H} \\
\mathbf{h}_{m} & =\mathbf{h}^{(\mathbf{v})} \cdot \mathbf{W}^{H} \mathbf{I}_{K \cdot n_{T}} \\
\Rightarrow \Phi & =\mathbf{R}_{\mathbf{h h}}^{-1} \mathbf{h}_{m},
\end{aligned}
$$

with $m=1, \ldots, K$. The solution is efficiently calculated by Cholesky decomposition of the covariance matrix $\mathbf{R}_{\mathbf{h h}}$. Adding the identity matrix is for numerical stabilisation only (Haykin, 2001). Finally, the filter matrix is given by

$\Psi=\mathbf{W}^{H} \Phi^{H}$.

Applying the filter to the received signals the estimated $n_{T}$ send signals for one OFDM symbol in time domain are calculated by $\tilde{\mathbf{s}}^{(\mathbf{v})}=\Psi \cdot \mathbf{r}^{(\mathbf{v})}$. The complexity is $O\left(K^{3} \cdot n_{R} \cdot n_{T}^{2}\right)$. It is obviously higher than in the frequency domain case because of the $K^{3}$ dependence. However, for large systems approximations can reduce the complexity as shown by Hueske and Götze (2009).

\section{Simulation results}

First, results are presented for the frequency domain equaliser algorithms. A $4 \times 4$ system was considered with Rayleigh block fading. Uniform distributed bits were LDPC coded with an irregular LDPC code with design code rate of $1 / 2$, interleaved and 4-QAM modulated. LDPC codes are chosen because of high codeword distance and parallelisable decoder structure (Richardson et al., 2001). BER versus SNR for the uncoded system are depicted in Fig. 3, for the decoded case see Fig. 4. For the decoding the equalised symbols were LLR demapped per layer, which reduces complexity with respect to the joint detection. In case of uncoded transmission, there are approximate four orders of magnitude between the optimal detection algorithms based on maximum likelihood and the linear approaches (ZF, MMSE) at $22 \mathrm{~dB}$. ZF and the MMSE approach show different behaviours for the coded 


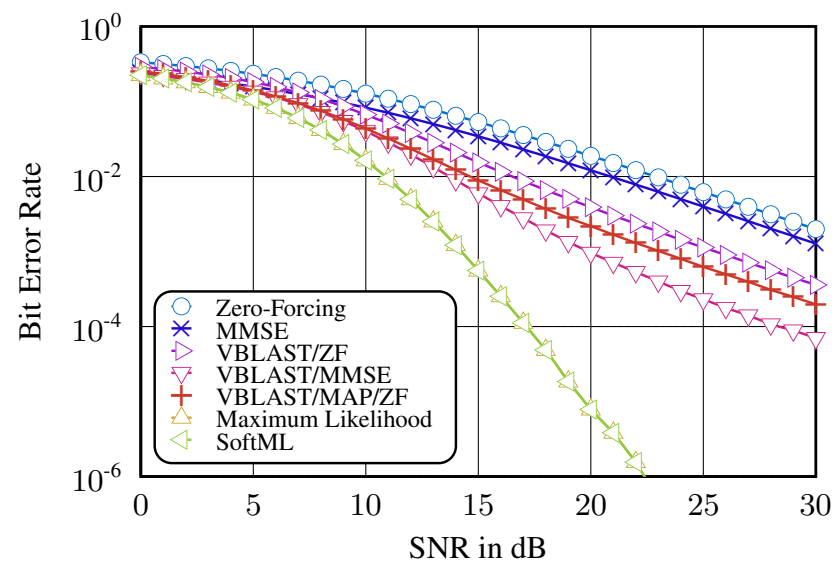

Fig. 3. BER vs. SNR for uncoded modulation over a Rayleigh block fading $4 \times 4$ MIMO channel and a selection of frequency domain equaliser.

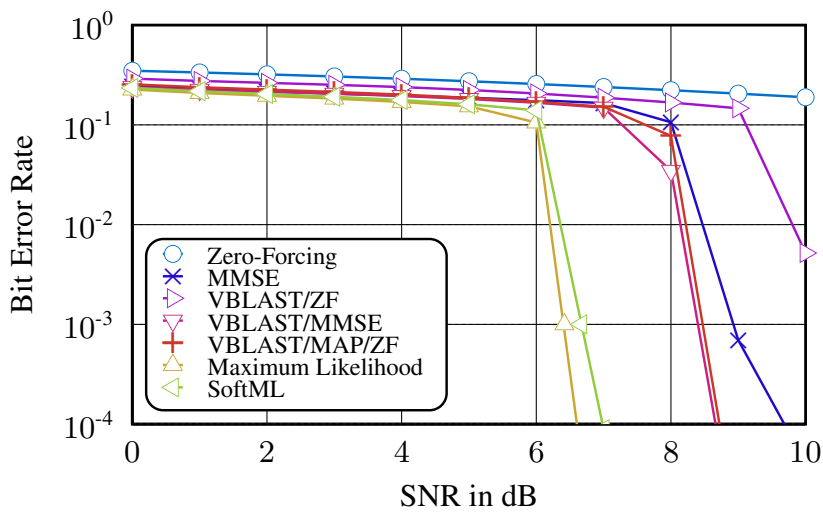

Fig. 4. BER vs. SNR for LDPC-coded bit-interleaved modulation over a Rayleigh block fading MIMO channel and the frequency domain equaliser from Fig. 3.

case. Comparison of VBLAST/ZF and VBLAST/MMSE demonstrates that neglecting noise leads to a significant performance loss. In the MMSE approach through the LLRs the uncertainty of an equalised symbol is carried to the decoder and can be reflected there. The simulations for comparison of FEQ resp. TEQ were performed on a $4 \times 4$ MIMO-OFDM system with $K=128$ subcarriers and a cyclic prefix length $L=6$. For a realistic channel the 3GPP Spatial Channel Model was used. The results are shown in Fig. 5. Note that for longer OFDM symbols e.g. $K^{\prime}=1024$ the normalised Doppler frequency increases with a factor 8 , so results for the velocity region of $160 \mathrm{~m} / \mathrm{s}$ would translate into the region around $20 \mathrm{~m} / \mathrm{s}$. It can be seen that for high velocities of the mobile station TEQ shows significant gains in terms of symbol error rate (SER) which increases with growing SNR.

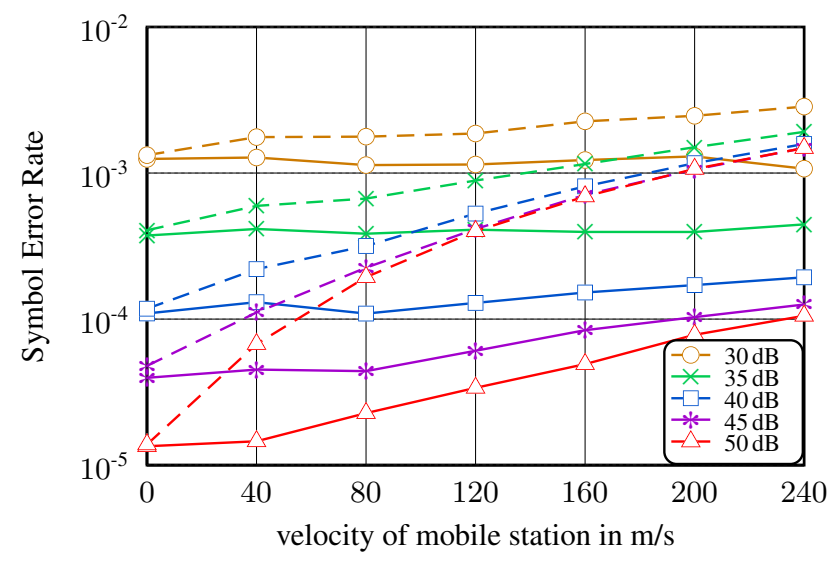

Fig. 5. Comparison of Time and Frequency Domain Equalisation performance TEQ (solid lines) resp. FEQ (dashed lines) for increasing Velocities and in high SNR regions, FEQ-method Zero-Forcing.

\section{Conclusions}

In this paper we have compared sub-optimal frequency domain equaliser with respect to coded performace in per layer LLR calculation and presented results for time domain equalisation mitigating intercarrier interference and spatial interference as well. We compared those results with the analogue equaliser in the frequency domain. For highly mobile terminals significant gains in terms of BER can be realized by employing time domain processing. When considering complexity, frequency domain equalisation is in favor, of course. To use those gains, future applications might switch to time domain equalisation at a certain velocity, depending on overall link quality and quality of service constrains.

\section{References}

3rd Generation Partnership Project (3GPP): Spatial channel model for Multiple Input Multiple Output (MIMO) simulations, Tech. Spec. Group Radio Access Network, Tech. Report 25.996, Rel. 8.0.0, 2008.

Haykin, S.: Adaptive Filter Theory, Prentice Hall, 4. edn., 2001.

Hochwald, B. and ten Brink, S.: Achieving near-capacity on a multiple-antenna channel, IEEE Trans. Commun., 51, 389-399, doi:10.1109/TCOMM.2003.809789, 2003.

Hueske, K. and Götze, J.: Ov-OFDM: A reduced PAPR and cyclic prefix free multicarrier transmission system, in: ISWCS 2009, 206-210, doi:10.1109/ISWCS.2009.5285357, 2009.

Richardson, T. J., Shokrollahi, M. A., and Urbanke, R. L.: Design of capacity-approaching irregular low-density parity-check codes, IEEE Trans. Inf. Theory, 47, 619-637, doi:http://dx.doi.org/10. 1109/18.910578, 2001.

Stamoulis, A., Diggavi, S., and Al-Dhahir, N.: Intercarrier interference in MIMO OFDM, IEEE Trans. Signal Process., 50, 24512464, doi:10.1109/TSP.2002.803347, 2002. 
Wolniansky, P. W., Foschini, G. J., Golden, G. D., and Valenzuela, R. A.: V-BLAST: An Architecture for Realizing Very High Data Rates Over the Rich-Scattering Wireless Channel, Proc. ISSSE, 295-300, 1998.
Yapici, Y.: V-Blast/MAP: A New Symbol Detection Algorithm for MIMO Channels, Master Thesis, Depart. of Electrical and Electronics Eng., Bilkent University, 2005. 